\title{
Probing Molecular Dynamics at Attosecond Resolution with Femtosecond Laser Pulses
}

\author{
X. M. Tong, ${ }^{*}$ Z. X. Zhao, and C. D. Lin \\ Physics Department, Kansas State University, Manhattan, KS 66506-2601
}

\begin{abstract}
The kinetic energy distribution of $\mathrm{D}^{+}$ions resulting from the interaction of a femtosecond laser pulse with $\mathrm{D}_{2}$ molecules is calculated based on the rescattering model. From analyzing the molecular dynamics, it is shown that the recollision time between the ionized electron and the $\mathrm{D}_{2}^{+}$ion can be read from the $\mathrm{D}^{+}$kinetic energy peaks to attosecond accuracy. We further suggest that more precise reading of the clock can be achieved by using shorter fs laser pulses (about $15 \mathrm{fs}$ ).
\end{abstract}

PACS numbers: 34.50.Rk, 31.70.Hq, 95.55.Sh

Human experience shows that new areas of science and technology open up with the ability to make measurements at increasingly shorter time regime. With the advent of femtosecond (fs) lasers, femtochemistry became possible where chemical reaction dynamics can be probed at the atomic scale [1]. Clearly, fs lasers cannot be used directly to probe electron dynamics which is in the attosecond (as) regime. While a substantial effort is being dedicated to developing single attosecond pulses [2, 3, 4, 5], presently few laboratories have such lasers available.

An ingenious suggestion for performing measurements at attosecond resolution with fs lasers was proposed by Corkum and his group recently. Their results were reported in two recent publications, here to be called I [6] and II []], respectively. In their experiment, a $40 \mathrm{fs}$ pulse, with mean wavelength ranging from $800 \mathrm{~nm}$ to $1850 \mathrm{~nm}$, and peak intensity of about $1.5 \times 10^{14} \mathrm{~W} / \mathrm{cm}^{2}$, was used to ionize a $\mathrm{D}_{2}$ molecule to produce $\mathrm{D}^{+}$ion. It was assumed that $\mathrm{D}_{2}$ was first ionized near the peak of the laser pulse to create a correlated electronic and nuclear wave packet. Within a single optical cycle, the electron was driven back to collide with $\mathrm{D}_{2}^{+}$and to excite it to the excited $\sigma_{u}$ electronic state which subsequently dissociated to produce $\mathrm{D}^{+}$. The kinetic energy of the $\mathrm{D}^{+}$ion reflects the internuclear distance, as well as the time when the rescattering occurs. With proper laser intensity, both the initial ionization and the rescattering are found to occur at time interval of far less than one optical cycle, thus providing attosecond temporal resolution, irrespective of the femtosecond pulse duration of the laser. In II, by changing the wavelength of the fs laser, they concluded that the dissociation dynamics of $\mathrm{D}_{2}^{+}$can be used as a molecular clock and the clock can be read with attosecond resolution.

To read the molecular clock accurately, the rescatter-

${ }^{*}$ Contact: xmtong@phys.ksu.edu ing mechanism which leads to the measurable $\mathrm{D}^{+}$kinetic energy distribution has to be understood in details. In this Letter we report the main conclusion of our careful analysis of the rescattering mechanism. In contradiction to I and II, our analysis shows that the $\mathrm{D}^{+}$ions are not produced by the dissociation of the excited $\mathrm{D}_{2}^{+}$ions. We found that the excited $\mathrm{D}_{2}^{+}$ions are readily further ionized by the laser and thus the $\mathrm{D}^{+}$ions measured in I and II are mostly coming from the Coulomb explosion along the $\mathrm{D}^{+}+\mathrm{D}^{+}$potential curve. Our conclusion is consistent with the recent experiment of Alnaser et al. [8] where the branching ratio of $\mathrm{D}^{+}$produced from Coulomb explosion vs. from dissociation was determined. Our analysis also shows that the dominant peak of the $\mathrm{D}^{+}$kinetic energy distribution is from the third return of the rescattering process, rather than from the first return, as assumed in I and II. This has the effect that the previous molecular clock was not read correctly. From our analysis we further suggest that a more precise reading of the clock can be achieved with a shorter fs pulse.

In Fig. 1 we depict the physical processes in the rescattering mechanism which are used for reading a "molecular clock". Similar to the experimental arrangement of I and II, the $\mathrm{D}_{2}$ molecules are aligned perpendicular to the direction of a linearly polarized infrared laser. This configuration eliminates $\mathrm{D}^{+}$ions from being produced either through bond softening or through charge resonance enhancement ionization [9, 10], even though both processes produce $\mathrm{D}^{+}$ions at lower kinetic energies which can be separated from the higher energy ions produced through the rescattering process [8] considered here. Following Fig. 1 , a $D_{2}$ molecule is ionized near the peak field of the laser pulse at $t_{0}$. This ionization launches two correlated wave packets: an electronic wave packet which is driven by the combined laser field and the Coulomb field of the $\mathrm{D}_{2}^{+}$ion, and a vibrational nuclear wave packet which is assumed to propagate freely in the $\sigma_{g}$ ground potential of $\mathrm{D}_{2}^{+}$. The vibrational wave packet at $t_{0}$ is taken to be the ground vibrational state of $\mathrm{D}_{2}$, assuming Frank-Condon 


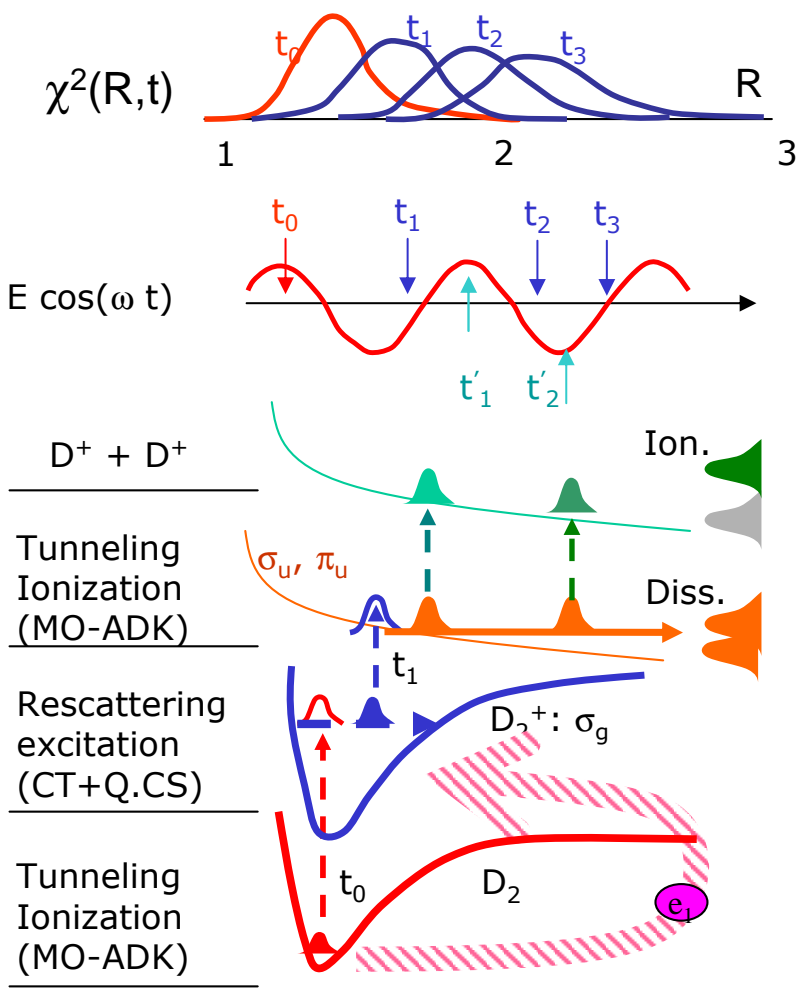

FIG. 1: Schematic of the major physical processes leading to the formation of $\mathrm{D}^{+}$ion. The $\mathrm{D}_{2}$ is first ionized at $t_{0}$ creating an electron wavepacket which returns to collide with $\mathrm{D}_{2}^{+}$at time $t_{1}$. In the meanwhile the initial vibrational wave packet, measured by $\chi^{2}(\mathrm{R}, \mathrm{t})$, created at $\mathrm{t}_{0}$, is shifted to larger $\mathrm{R}$ and broadened at later time. At $\mathrm{t}_{1}$, the $\mathrm{D}_{2}^{+}$is excited from $\sigma_{g}$ to $\sigma_{u}$ and $\pi_{u}$ by electron impact. The excited $\mathrm{D}_{2}^{+}$can dissociate directly to give $\mathrm{D}^{+}$, or can be further ionized at $\mathrm{t}_{1}{ }_{1}, \mathrm{t}_{2}{ }_{2}$, etc to produce two $\mathrm{D}^{+}$ions by Coulomb explosion. Note that similar rescattering processes can be initiated at later time, $\mathrm{t}_{2}, \mathrm{t}_{3}$, etc, and are included in the calculated $\mathrm{D}^{+}$spectra.

principle. For $t>t_{0}$, the vibrational wave packet propagates outward and broadens, as shown in the top frame of Fig. 1. The electron initially is driven out by the laser field but returns after $2 / 3$ of an optical cycle to recollide with $\mathrm{D}_{2}^{+}$at time $\mathrm{t}_{1}$. The rescattering can excite $\mathrm{D}_{2}^{+}$to higher electronic states or to ionize it. To simplify the operation of the clock, the laser intensity is chosen such that the returning electron has small energy and ionization is negligible. Once the $\mathrm{D}_{2}^{+}$is in the excited electronic state, it can dissociate or it can be further ionized by the laser field. Since the laser pulse lasts for many optical cycles, the electron can revisit the $\mathrm{D}_{2}^{+}$ion many cycles later after the initial ionization. Thus rescattering can occur on the second return at $t_{2}$, on the third return at $t_{3}$, etc, see Fig. 1. For each initial $t_{0}$, the return times $t_{1}, t_{2}, t_{3}$, $\ldots$ are fairly well defined. Since the initial ionization at $t_{0}$ occurs only in a sub-fs time interval, the rescattering times $\mathrm{t}_{i}(\mathrm{i}=1,2,3, \ldots)$ are also defined at the sub-fs (or attosecond) accuracy. If the rescattering populates only the lowest excited electronic state, $\sigma_{u}$, and then the $\mathrm{D}_{2}^{+}$ dissociates, the peaks of the kinetic energy released can be used to read the clock at the rescattering times, as depicted in Fig. 1.

We have examined the rescattering model in details and found three major modifications to the simple model used in I and II. First, we found that excitation to the excited $\pi_{u}$ electronic state is not negligible in general. Second, once the $\mathrm{D}_{2}^{+}$ions are in the excited electronic states, they are readily ionized by the laser fields such that the $\mathrm{D}^{+}$ions are predominantly produced from the Coulomb repulsion. This modifies the $\mathrm{D}^{+}$ion energy distributions. Third, we found that rescattering from the third return is more important than from the first return, in disagreement with the model of I and II. However, our conclusion is identical to the rescattering model results of He where multiple returns have been shown to be dominant [1].

In the modelling, we calculate the ionization rate at $t_{0}$ using the molecular tunneling (MO-ADK) model for a $\mathrm{D}_{2}$ molecule aligned perpendicular to the laser polarization 12. The subsequent trajectory of the ionized electron is calculated classically in the field of $\mathrm{D}_{2}^{+}$and the laser, with initial longitudinal and transverse velocity distributions determined according to the ADK theory. The return energy and return time of the electron at the distance of closest approach are calculated. To obtain electron impact excitation probability from $\sigma_{g}$ to the excited $\sigma_{u}$ and $\pi_{u}$ electronic states of $\mathrm{D}_{2}^{+}$, we used scaling relation similar to that used for He 11], taking the 1s to $2 p$ excitation cross section for electron impact on $\mathrm{H}$ and $\mathrm{He}^{+}$ as input data [13]. Since $\mathrm{D}_{2}^{+}$is aligned perpendicular to the electron beam direction, we use the $2 \mathrm{p}_{1}$ excitation cross section for the $\sigma_{u}$ state and the $2 \mathrm{p}_{0}$ excitation cross section for the $\pi_{u}$ state, but each rescaled with their respective excitation energies. It is interesting to note that electron impact excitation to $2 \mathrm{p}_{0}$ is five to three times larger than excitation to $2 \mathrm{p}_{1}$ in the energy region of interest (the branching ratio for data from Bray [14] for $\mathrm{H}$ and from the experimental data of [15] for He are essentially identical). Thus, different from I and II, we found that excitation to $\pi_{u}$ state is not negligible. Once the $\mathrm{D}_{2}^{+}$ ions are in the excited electronic states, they can dissociate or further ionized by the laser. We use the MO-ADK model 12] to calculate the ionization rate of $\mathrm{D}_{2}^{+}$in the $\sigma_{u}$ and $\pi_{u}$ states, at different internuclear separations and for molecules aligned perpendicular to the laser polarization. Since the electron cloud of the $\pi_{u}$ state for the perpendicularly aligned $\mathrm{D}_{2}^{+}$is along the laser field direction, it is readily ionized. For the $\sigma_{u}$ the ionization rates are smaller but increase rapidly with laser intensity. Using the ionization rates which are large only near $t_{1}^{\prime}, t_{2}^{\prime}$, ... (see Fig. 1) following the excitation of $\mathrm{D}_{2}^{+}$at $t_{1}, t_{2}$, etc, we calculated the ionization and dissociation yield and the $\mathrm{D}^{+}$kinetic energy distributions. Since the vibrational wave packet spreads as time increases, we need to weight the R-dependence at each time in calculating the ionization and dissociation yields, as well as the cor- 


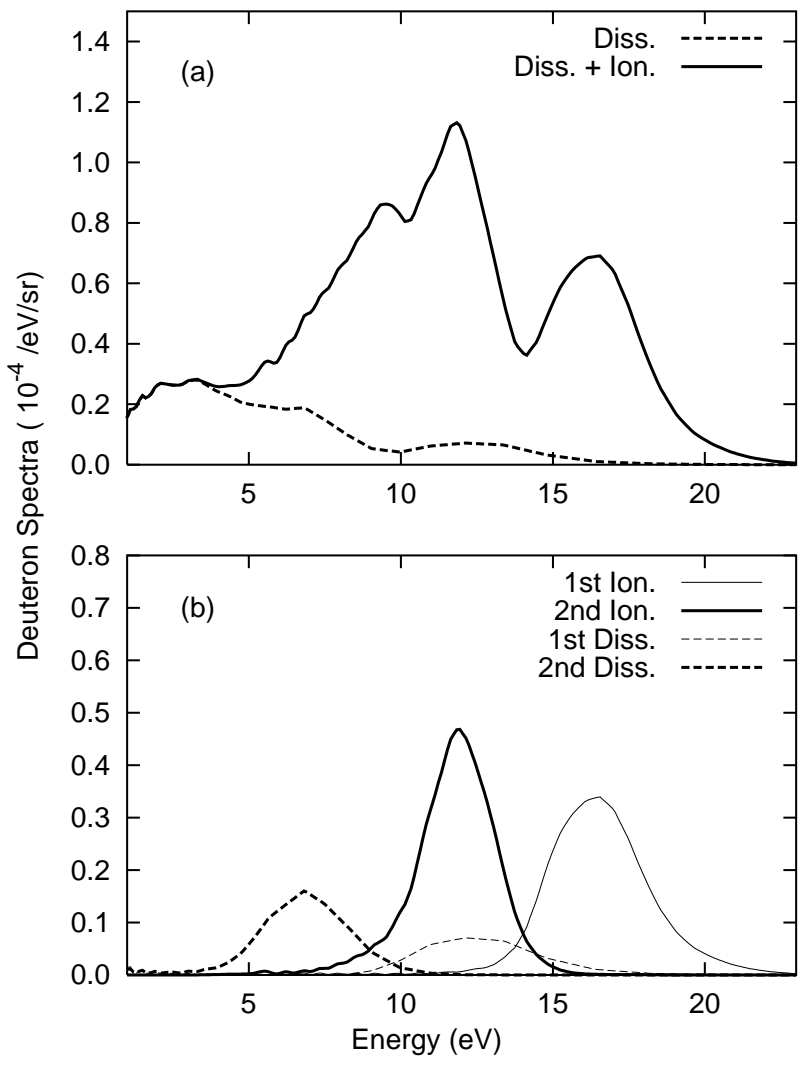

FIG. 2: (a) The $\mathrm{D}^{+}$kinetic energy spectra predicted by the present rescattering model. The dashed lines give contribution from dissociation alone. (b) Solid lines: $\mathrm{D}^{+}$spectra from the ionization channel, from the rescattering in the first (higher energy peak) and 2nd optical cycles (lower energy peak) after the initial ionization of $\mathrm{D}_{2}$. Dashed lines, the same except from the dissociation. Laser parameters used: Peak intensity is $1.5 \times 10^{14} \mathrm{~W} / \mathrm{cm}^{2}$, pulse length is $40 \mathrm{fs}$ and mean wavelength is $800 \mathrm{~nm}$.

responding energy of $\mathrm{D}^{+}$. In the simulation, we also have to integrate $t_{0}$ near the peak of the field where ionization of $\mathrm{D}_{2}$ was initiated.

Figure 2(a) shows the total $\mathrm{D}^{+}$spectra obtained from the present rescattering model. The total yield from ionization and dissociation is shown in solid line. Note that the dissociation alone, shown in dashed lines, contributes little to the ion yield, except at lower energies. In Fig. 2(b) we identify the dissociation and ionization yields from rescattering at different times. This is important since it determines how precisely a molecular clock can be read. In the figure we separate the contributions into 1st and 2nd cycles. Each cycle is defined to be one full optical cycle after the electron is born. Thus the ionization peak from the first cycle measures $\mathrm{D}^{+}$ion from rescattering occurring near $t_{1}$, while the ionization yield from the second cycle is due to rescattering occurring mostly at $t_{2}$ and $t_{3}$. Since the return energy at $t_{2}$ is smaller, contribution in the second cycle comes mostly from rescattering at $t_{3}$. From Fig. 2(b), the peak posi- tion for the 3rd return in the ionization spectra essentially coincides with the peak position from the first return in the dissociation spectra. This peak in the total $\mathrm{D}^{+}$ion spectra was identified in II as a reading of the clock at $t_{1}$, while our analysis shows that this should be a reading of $t_{3}$. (Note that the spectra in Fig. 2) (a) include contributions from higher returns that are not shown in Fig. 2(b).)

In Fig. 3(a) we show the calculated $\mathrm{D}^{+}$spectra at four different wavelengths used in II. The data are to be compared to their Fig. 2 except that we plot against the sum energy which is twice the energy per $\mathrm{D}^{+}$used in II. The comparison shows reasonable agreement, but not in details, since the modelling does not account for the volume effect and the finite acceptance angle used in the experiment. However, the shift of the main peak with increasing wavelength is clear. In Table \we compare the data deduced from II with the data deduced from our calculations shown in Fig. 3(a). The experimental peak energy for each wavelength is shown in column 2. Assuming that the peak is due to dissociation for rescattering at $t_{1}$, the expected kinetic energy, the mean internuclear separation and the rescattering time $t_{1}$ were derived in

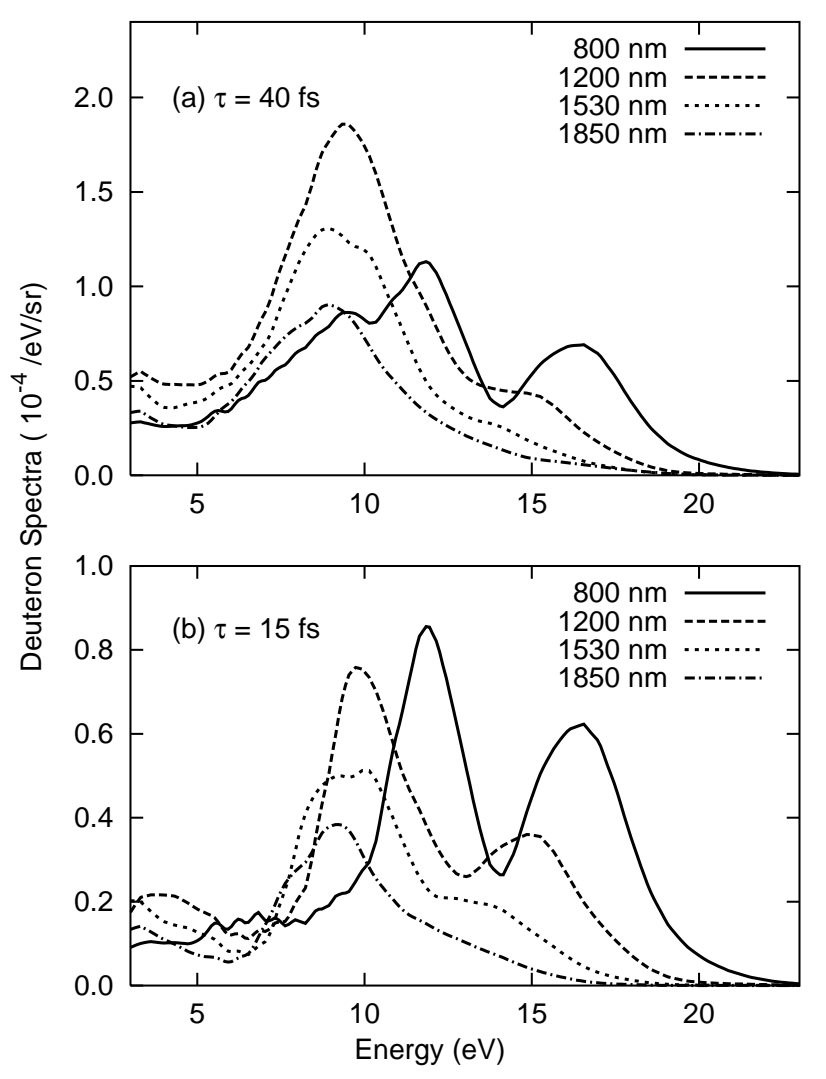

FIG. 3: (a) The dependence of the $\mathrm{D}^{+}$kinetic energy spectra on the mean laser wavelength. The peak from the first return is clearly visible only for the $800 \mathrm{~nm}$ pulse. Laser peak intensity is $1.5 \times 10^{14} \mathrm{~W} / \mathrm{cm}^{2}$ and pulse length is $40 \mathrm{fs}$. (b) Same as (a) except that the pulse length is reduced to $15 \mathrm{fs}$. 
TABLE I: Comparison of the $\mathrm{D}^{+}$kinetic energy peak positions $(\mathrm{E}$ in $\mathrm{eV})$, and the deduced internuclear separation $(\mathrm{R}$, in a.u.) and the time ( $t$, in $f s)$ where rescattering occurs. The experimental data and model results quoted in II [7] are shown together with the predictions from the present work.

\begin{tabular}{||r||r||r|r|r||r|r|r||}
\hline \hline \multicolumn{1}{|||}{} & \multicolumn{1}{|c||}{ Exp. (II) } & \multicolumn{3}{c||}{ Model (II) } & \multicolumn{3}{|c||}{ This Work } \\
\hline$\lambda(\mathrm{nm})$ & $\mathrm{E}$ & $\mathrm{E}$ & $\mathrm{R}$ & $\mathrm{t}_{1}$ & $\mathrm{E}$ & $\mathrm{R}$ & $\mathrm{t}_{3}$ \\
\hline 800 & 12.0 & 12.6 & 1.68 & 1.7 & 12.0 & 2.27 & 4.4 \\
1200 & 10.0 & 10.7 & 1.84 & 2.6 & 9.4 & 2.90 & 6.7 \\
1530 & 8.6 & 9.7 & 1.94 & 3.3 & 8.7 & 3.12 & 8.6 \\
1850 & 8.0 & 8.1 & 2.10 & 4.4 & 8.2 & 3.30 & 10.5 \\
\hline \hline
\end{tabular}

II, and are shown in the third column. According to our model, the peak at each wavelength is due to rescattering at $t_{3}$, obtained from the ionization signal. The predicted deuteron energies from our modelling, shown in the 4th column, are in good agreement with the experimental data. However, we read a different mean internuclear separation and a different rescattering time. From this Table, we note that these measurements imply a subangstrom resolution in the internuclear separation or an attosecond resolution in the recollision time.

In Fig. 3(a) the peak positions for the $1530 \mathrm{~nm}$ and $1850 \mathrm{~nm}$ data points are not very sharp. From Table I we note that $t_{3}$ for each case is 8.6 fs and 10.5 fs respectively. At such long time after the wave packet is created, the spreading of the vibrational wave packet is large such that the clock can no longer be well read. In fact, the peak energy and the mean internuclear separation for these two wavelengths are calculated from the third return alone, not from the full spectra. Thus the longer wavelength laser blurs the reading of the clock.

Another issue that affects the reading of the clock is the pulse duration. A clock designed to read $t_{1}$ appears preferable since the vibrational wave packet is not significantly broadened within one optical cycle. However, at $t_{1}$ the mean internuclear separation is small and the molecular potential curves are steep. Upon reflection, this results in a rather broad $\mathrm{D}^{+}$kinetic energy spectrum, see the dissociation or ionization peaks from the first return in Fig. 2(b). Signals from the first return are also weak, and the structures are easily buried by contributions from the third return. Indeed, our model indicates that the kinetic energy spectra are dominated by rescattering from the third return. Even though the vibrational wave packet is broadened more at $t_{3}$, the breakup occurs at larger internuclear separations where the potential curves are rather flat, resulting in sharper deuteron energy spectra, see Fig. 2(b). Unfortunately for a 40 fs pulse used in I and II, higher returns ( for $\left.t>t_{3}\right)$ still contribute to the rescattering process. These higher returns modify the ion yield in the lower energy region, thus shifting the overall peak position. By using a shorter laser pulse, contributions from these higher multiple returns can be eliminated. In Fig. 3(b) we show the calculated ion yield for a 15 fs laser pulse at the same intensity as in Fig. 3(a). Clearly the main peak in the ion yield is more prominent and thus the time resolution is improved. Note that the clock is not expected to be affected by the lack of knowledge of the absolute phase of the few-cycle laser pulse since the clock is measured from the time $t_{0}$ where $D_{2}$ is first ionized. However, our analysis does not include volume effect, nor the acceptance angle of $\mathrm{D}^{+}$ions, which can reduce the precision of the molecular clock.

In summary, we examined the rescattering mechanism for the production of high energy $\mathrm{D}^{+}$ions in laser- $\mathrm{D}_{2}$ interaction. We show that the $\mathrm{D}^{+}$ions are produced mainly not from the dissociation of $\mathrm{D}_{2}^{+}$after it is excited by the returning electron, but rather by Coulomb explosion after the excited $\mathrm{D}_{2}^{+}$is further ionized by the laser. We agree with Niikura et al. [6, 7] that the ion spectra can be used as a molecular clock with attosecond precision, and suggest that experiments with a shorter fs (about 15fs) laser pulse would improve the precision of the clock.

This work is in part supported by Chemical Sciences, Geosciences and Biosciences Division, Office of Basic Energy Sciences, Office of Science, U. S. Department of Energy. CDL also wishes to thank Igor Bray for communicating to him the partial $1 \mathrm{~s} \rightarrow 2 \mathrm{p}_{m} \quad(\mathrm{~m}=0,1)$ cross sections.

[1] A. H. Zewail, J. Phys. Chem. A 104, 5660 (2000).

[2] M. Drescher et al., Science 291, 1923 (2001).

[3] M. Hentschel et al., Nature 414, 509 (2001).

[4] M. Drescher et al., Nature 419, 803 (2002).

[5] A. Baltuska et al., Nature 421, 611 (2003).

[6] H. Niikura et al., Nature 417, 917 (2002).

[7] H. Niikura et al., Nature 421, 826 (2003).

[8] A. Alnaser et al., Phys. Rev. Lett. (2003), (in press).

[9] Y. Zou et al., Phys. Rev. A 51, 3790 (1995).

[10] A. D. Bandrauk and J. Ruel, Phys. Rev. A 59, 2153 (1999).

[11] G. L. Yudin and M. Y. Ivanov, Phys. Rev. A 63, 033404 (2001); 64, 035401 (2001).

[12] X. M. Tong, Z. X. Zhao, and C. D. Lin, Phys. Rev. A 66, 033402 (2002).

[13] I. Bray, CCC-database, http://atom.murdoch.edu.au/CCC-WWW/index.html

[14] I. Bray (private communication).

[15] H. Merabet et al., Phys. Rev. A 60, 1187 (1999). 